Chapter 4

\title{
Assisted Cycling Therapy for Persons with Down Syndrome - Implications for Improvements in Cognitive Functioning
}

\author{
Shannon D. R. Ringenbach, Simon Holzapfel, Genna M. Mulvey and \\ Sachin Pandya
}

Additional information is available at the end of the chapter

http://dx.doi.org/10.5772/60636

\begin{abstract}
To date, there are few, if any, behavioral or exercise interventions that have been shown effective in improving cognitive functioning in adolescents with Down syndrome(DS). Exercise is a logical answer because it has been repeatedly shown to improve cognitive and physical and mental health in typical populations. However, current exercise recommendations for persons with DS vary greatly. Recommendations are often nonspecific in terms of the type or intensity of exercise, and results on improvement of cognitive functioning are equivocal. This chapter will report on preliminary data of an 8 week intervention of assisted versus voluntary cycling exercise on cognitive and health functions in adolescents with DS. Assisted Cycling Therapy (ACT) is innovative and important because it is predicted to enhance neurogenesis, which in turn may improve multiple central nervous system functions related comorbid conditions in adolescents with DS.
\end{abstract}

Keywords: Executive function, aerobic exercise, activities of daily living, Alzheimer's disease, brain imaging

\section{Introduction}

Down syndrome (DS) is one of the most prevalent chromosomal conditions, affecting 1 in every 691 live births in the U.S.A. [1]. One of the main features of DS includes cognitive impairments. 
Specifically, adolescents with DS have been shown to have lower levels on executive functions including working memory, inhibition, planning and set switching than typically developing children matched for mental age [2]. These cognitive deficits can limit their abilities to perform activities of daily living. Thus, interventions to improve their capacity to perform activities independently would help to improve quality of life and reduce the costs associated with providing care for them. We are investigating the effects of physical activity interventions on the cognitive skills in persons with DS.

\subsection{Executive function}

Executive functions are a set of higher-order control processes that take place primarily in the frontal lobe of the brain [2], which deal with the decisions to make actions, and planning how to accomplish tasks [3]. Executive function includes concept formation, task switching, inhibition, volition, planning, purposeful action, and effective performance [3]. These are necessary in order for a person to engage in tasks independently. People with deficits in executive function are often called lazy due to this lack of initiative, but executive function is necessary for a person to initiate self-care routines or work independently. Many researches have documented that people with DS have shown deficits in executive functioning (e.g., [4, 5]). Improving executive functions could in turn improve many other independent living skills. Below we have highlighted a few executive functions that we measured in response to an exercise intervention in persons with DS.

\subsection{Working memory}

Working memory is information that people actively keep in their mind and manipulate [3, 6]. If human memory were a computer, the working memory would have been an active window where a person would have manipulated the informational contents. Working memory is limited in size, yet it is important for many other tasks from remembering words to learning new motor skills [6,7]. Several studies have found that people with DS have significant deficits in working memory $[8,9]$.

\subsection{Set switching}

Set switching is the ability to change a course of thought or action based on changing requirements [3]. In clinical settings, this is typically done with a card sorting test where children are first asked to sort the cards by shape and then by color. Children with typical development are unable to switch to the second sorting rule at three years old. By four years of age, a child can change rules with some struggle, and by five years old, a child can shift to the new rule with ease [10]. On a practical level, set switching is demonstrated while children are working on something when a parent tells them that they need to get ready to leave the house. The ease at which the children are able to transit between the two tasks reflects their capacity for set switching. Set switching also requires working memory to process the change in tasks and the ability to inhibit the first behavior pattern [11]. Set switching activates a network of cells in the frontoparietal region of the brain, including the inferior frontal gyrus, anterior cingulate cortex, and supramarginal gyrus [12]. When someone has difficulty with set switching, it can result 
in inflexibility and perseverative behaviors. People with DS have significant deficits in set switching in comparison to people with typical development $[2,8]$.

\subsection{Verbal fluency}

People with DS in general show deficits with language, especially with expressive vocabulary [13-15]. Typically this is tested by asking people to recall words related to a particular category or words that start with a certain letter. Neuroimaging studies have shown that letter-based verbal fluency is mediated by the frontal cortex and category-based verbal fluency by the temporal cortex; parietal lobe mediates both tasks [16]. Nash and Snowling [17] found that people with DS showed deficits in verbal fluency in comparison with peers of typical development.

As previously described, there is a vast amount of research that documents cognitive deficits in persons with DS. We believe that it is time to focus on interventions aimed at improving cognitive functions in persons with DS. Our innovative exercise intervention and results will be explained next.

\section{Intervention: Move fast, think fast}

Exercise is a logical intervention for effective treatment of cognitive impairments in persons with DS because the positive influence of voluntary exercise on cognition has been demonstrated in other typical populations [18, 19], including children [20,21] and older adults [22, 23]. Furthermore, voluntary exercise has been shown to improve memory in mice models (Ts65Dn) of DS [24]. However, a recent review of the therapeutic benefits of exercise in persons with DS found that exercise was nonsignificant in improving physical and mental health outcomes in persons with DS [25]. Because persons with DS move slowly [26] due to slower reaction times [27], deficits in muscular strength [28], and reduced cardiorespiratory capacity [29], adolescents with DS typically 'do' not exercise at a relatively high rate, 'thus, they miss out on the opportunity to gain' cognitive improvements through neuroplasticity in the brain. Furthermore, approximately $61 \%$ of persons with DS have been shown to have low exercise tolerance [30] which reduces their exercise time and intensity and which seems to limit the cognitive benefits of exercise for persons with DS [25]. The voluntary aspect of the exercise imposes major limitations in the quantity and quality of exercise in special populations such as those with DS.

There is an emerging body of literature in healthy older adults and individuals with Alzheimer's disease indicating that exercise results in structural and functional changes in the brain [31]. These alterations in brain structure and function suggest that CNS function can be altered via voluntary exercise in individuals with relatively normal and abnormal patterns of activation within the motor cortex. However, because persons with DS have limited motor output due to physiological and psychosocial factors, their ability to induce changes in CNS function may be compromised when engaging in voluntary exercise performed at their preferred (i.e., low) rates. They may need to have exercise augmented through mechanical assistance as 
proposed in our assisted exercise paradigm, coined Assissted Cycling Therapy (ACT) in 2013 [32]. Assisted exercise is an approach initially used with animals which were exercised on a motorized treadmill at a rate greater than their voluntary exercise rate. Assisted exercise has demonstrated improvements in cognitive functioning in animals [33] and most recently in patients with Parkinson's disease [34, 35]. ACT has been suggested to improve motor and cognitive function through its neuroprotective properties as demonstrated in Figure 1, a model proposed by Alberts and colleagues [34].

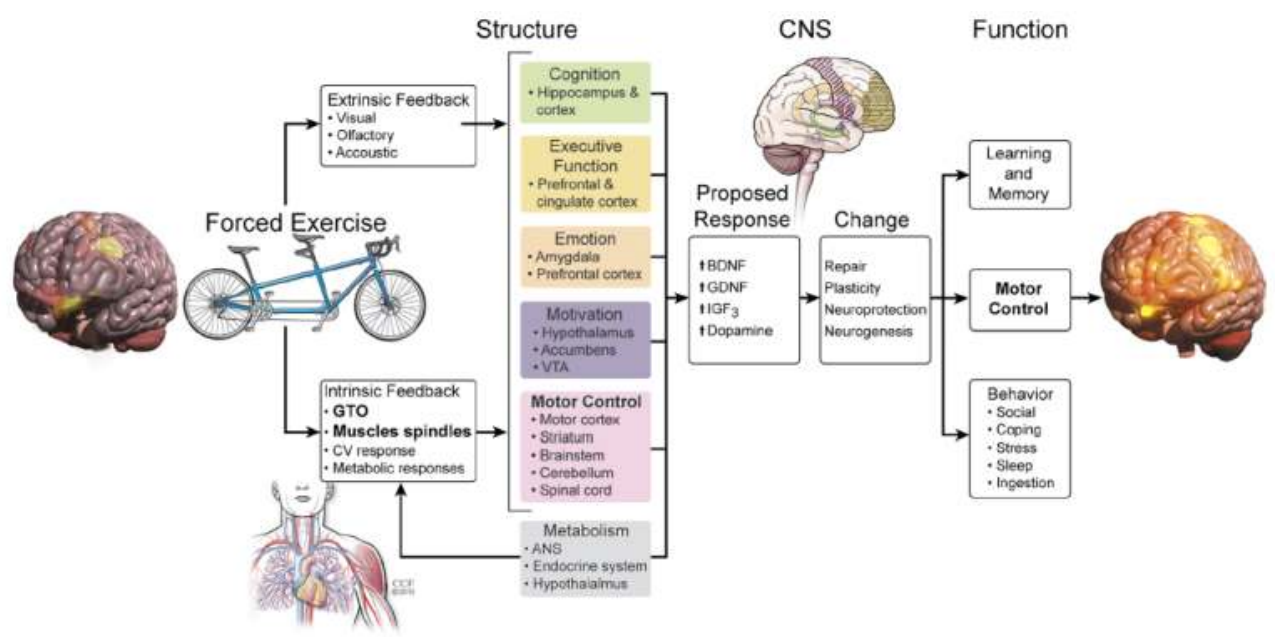

Figure 1. Proposed model for ACT (forced exercise) (Alberts et al., 2011).

\subsection{Procedures for assisted cycling therapy}

ACT is an emerging exercise paradigm especially suited for clinical populations who have limited voluntary movement output, exercise capacity, or exercise motivation. During ACT the electric motor of the bicycle is engaged which helps to increase pedaling cadence to a predetermined rate. We have used absolute cadences of approximately $80 \mathrm{rpm}$ or relative cadences of $35 \%$ greater than the initial voluntary pedaling rate. The initial pedaling rates, however, may need to be increased gradually for comfort and familiarization. The ACT condition often leads to reduced power compared to voluntary pedaling as indicated in Table 1 by the lower average power contribution of our participants in the ACT condition than the voluntary cycling condition. As can be seen in Figure 2, special procedures were utilized to ensure that the feet were not positioned too far forward and that they did not slip forward, side-to-side, or backward to ensure a high degree of safety at the high pedaling rates.

The length of our intervention period was eight weeks with three cycling sessions per week. Before each cycling session, the resting heart rate (HR) was obtained while the participant was sitting on the bike. A five-minute warm up at a voluntary rate was completed before the 30- 


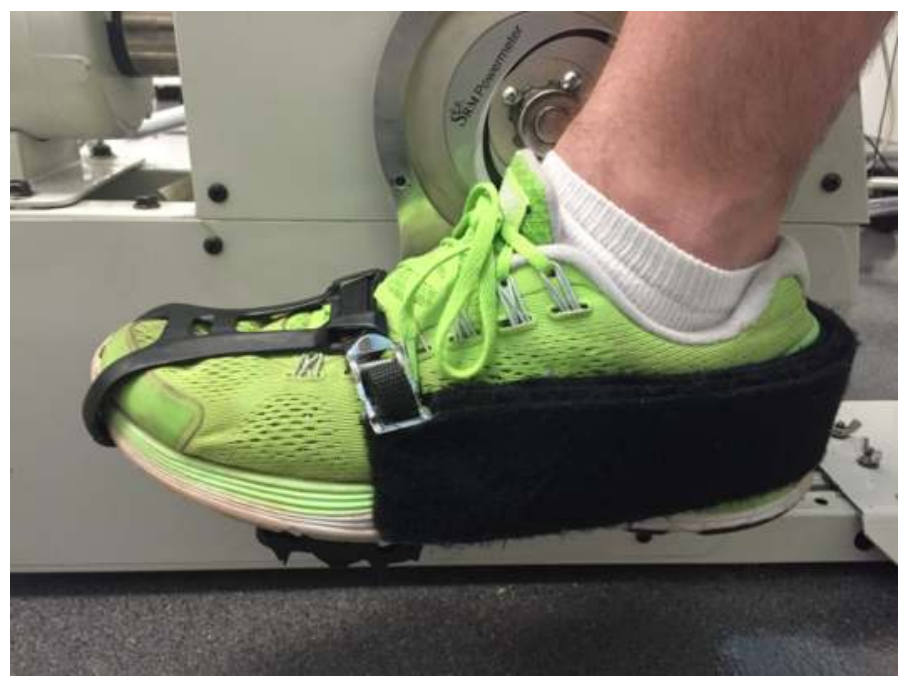

Figure 2. Pedal-foot interface.

minute cycling session regardless of the condition (ACT or voluntary cycling (VC)). On the first day, the average cadence from the warm-up period was multiplied by 1.35 to determine the initial ACT cadence. This step was omitted in the voluntary cycling condition. Thus, the cadence on the first day of the ACT intervention was set at a rate which was $35 \%$ faster than the voluntary cadence. A three- to five-minute cool-down at the end of the ACT or voluntary cycling session was optional. During the cool-down, the motor was not engaged. The average HR (bpm), cadence (rpm), and power (Watts) was recorded every five minutes during the cycling session (refer to Table 1 for mean values). These averages did not include the warmup period.

To monitor Rate of Perceived Exertion (RPE), we used a modified -point RPE scale. A rating of 2 or 3 on the -point RPE scale (1, easy/not-tired; 2, a little hard/a little tired; 3, hard/tired; 4, very hard/very tired) was desired to keep the exercise intensity at a moderate level. The goal was for participants to cycle between 64 and $76 \%$ of their age-predicted maximal HR (HRmax $=210-0.56 \times$ age -31 , [36]) which corresponds with a moderate exercise intensity as dictated by the American College of Sports Medicine [37]. Thus, for most participants in the ACT condition, we increased cadence from session to session by $3-5 \mathrm{rpm}$, based on tolerance, up to the maximum cadence of the motor (e.g., $95 \mathrm{rpm}$ ) or until $64 \%$ of age-predicted HRmax or a personal tolerance limit was reached. Participants in the ACT group took on average 13.2 cycling sessions to reach this point. Participants in the voluntary cycling group were not encouraged to pedal faster as the goal was to have them exercise at their preferred voluntary rate (refer to Table 1 for cadence values).

For this randomized control trial, participants were randomly allocated to eight weeks of ACT, eight weeks of VC, or eight weeks of no cycling (NC). The ACT and VC conditions were 
described in the previous section. Participants in the NC group completed only the pre- and posttesting sessions separated by eight weeks and they were instructed not to change their usual physical activity habits and therapy regiments for the eight weeks. Inclusion criteria consisted of trisomy-21 and a chronological age of 9-26 years. Exclusion criteria consisted of other genetic conditions and neurological disorders (e.g., ADHD and autism), medical contraindications to exercise, and sensory or physical impairments which preclude completion of the cycling intervention. During the pretesting sessions (first visit to the laboratory), the participants' height, weight, vision, hearing, and mental age were recorded or assessed. Mental age was determined with the Peabody Picture Vocabulary Test ( $4^{\text {th }}$ ed.; [38]) (refer to Table 1 for chronological andmental age values). In addition, all participants had functional hearing and vision for the purpose of the testing procedures. Then, three executive function tests were administered in random order.

\begin{tabular}{lllllllc}
\hline & \multicolumn{2}{l}{ ACT $(\mathbf{n}=\mathbf{1 8})$} & \multicolumn{2}{l}{ VC $(\mathbf{n}=\mathbf{1 6})$} & \multicolumn{2}{l}{ NC (n= 14) } & \multicolumn{2}{c}{ One-way ANOVA } \\
\cline { 2 - 6 } & Mean & SD & Mean & SD & Mean & SD & p-value \\
\hline Chronological age (years) & 19.4 & 4.9 & 18.4 & 3.4 & 17.0 & 4.0 & 0.304 \\
Mental age (years) & 6.1 & 3.3 & 5.2 & 2.1 & 6.0 & 1.8 & 0.687 \\
BMI $\left(\mathrm{kg} / \mathrm{m}^{2}\right)$ & 27.7 & 7.0 & 27.3 & 4.2 & 27.5 & 9.5 & 0.889 \\
Cadence (rpm) & 77.2 & 2.2 & 43.1 & 8.9 & & & $<0.001^{11}$ \\
Power (Watts) & 22.1 & 12.1 & 26.6 & 21.7 & & & $0.396^{11}$ \\
Heart rate (bpm) & 98.7 & 8.0 & 100.7 & 7.7 & & & $0.642^{11}$ \\
\hline
\end{tabular}

${ }^{1}$ Independent samples t-tests were used to test group differences.

Table 1. Descriptive statistics

\section{Measures}

The verbal fluency test consisted of four categories: animals, food and drinks, words that start with an S, and words that start with an F. The participants were given one minute per category and had to name as many words in the category as possible. The verbal fluency test was a test of verbal long term and working memory, attention, and inhibition [39, 40]. As mentioned, verbal fluency and other speech and language deficits are well documented in persons with DS [41-43]. Verbal fluency tests have been used as behavioral measures of hippocampal and prefrontal cortex function $[40,43]$.

A backward digit span test was administered as a behavioral measure of working memory, which requires the simultaneous storage and processing of information [6, 44]. It is considered a prefrontal function [6]. During the backward digit span test, participants had to reverse a sequence of numbers given by the investigator. The investigator was providing progressively longer sequences of numbers until the participant could no longer accurately articulate the given sequence in reverse order. 
The Wisconsin Card Sorting test (modified for DS) measures set switching ability and working memory which are functions of the frontal cortex and parts of the parietal lobe $[45,46]$. In this task, the participants are asked to match either shapes or colors with rule changes taking place during testing. Adolescents with DS have been found to have reduced capacity for set switching compared to typically developing adolescents [2]. These three executive function tests were repeated during posttesting.

\section{Results}

Cohen's $d$ effect sizes are considered small to medium if they range from 0.2 to 0.5 , medium to large if they range from 0.5 to 0.8 , and large if they are greater than 0.8 [47]. The effect sizes for the verbal fluency composite score were $d=0.15$ for ACT, $d=0.21$ for $\mathrm{VC}$, and $d=0.06$ for NC. The verbal fluency of the ACT and VC groups improved more than the verbal fluency of the NC group. The effect sizes for the backward digit span test were $d=0.31$ for the ACT group, $d=0.17$ for the VC group, and $d=0.00$ for the NC group. The ACT and VC groups both showed improved working memory while the improvement was greatest in the ACT group. The effect sizes for the Wisconsin Card Sorting test were $d=-0.21$ for the ACT group, $d=0.87$ for the VC group, and $d=0.00$ for the NC group. Only the VC group improved in their set-switching ability. See Figure 3 for a visual representation of the results.
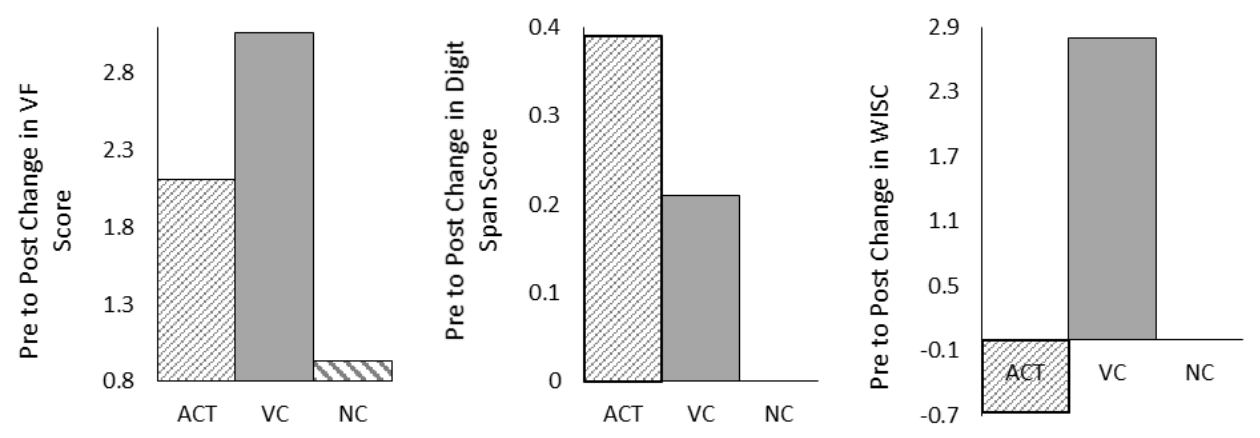

Figure 3. Pre- to postchange scores (postscore-prescore) in the verbal fluency test (VF), backward digit memory span test, and the Wisconsin Card Sorting test (WISC) by group. Larger, more positive change scores reflect an improvement in cognitive function.

\section{Interpretation of results}

It is clear that cycling exercise, whether it is assisted or voluntary, is more beneficial to executive function than no exercise. However, ACT seems to be more effective in improving working 
memory, whereas VC seems to be more effective in improving verbal long-term memory, and set switching than ACT.

Based on our results, a moderate exercise intensity, of between 64 and 76\% HRmax, may not be necessary for benefits as the average HR during ACT or VC cycling sessions was just below $64 \%$ of the average age-predicted HRmax. The average chronological age of our participants in the ACT and VC group was 19.4 years and 18.4 years, respectively. This translates to minimum average target HRs (64\%) of $107.6 \mathrm{bpm}$ and $108.0 \mathrm{bpm}$ in the ACT and VC group, respectively. In addition, their average exercising target $\mathrm{HR}$ of $98.7 \mathrm{bpm}$ and $100.7 \mathrm{bpm}$ were below the target HR range. In fact, on the first day of cycling, only $11 \%$ of ACT and $31 \% \mathrm{VC}$ participants reached $64 \%$ of their age-predicted maximal HR. The only difference between the ACT and VC groups was the cadence at which they were cycling. We can thus conclude that the specific adaptations in terms of executive function are due to the different rates of movement.

The greater movement frequency during ACT would presumably lead to more frequent stimulation of the Golgi tendon organs and muscle spindle fibers in the lower extremity musculature and associated tendons, which in turn translates to greater afferent input to the frontal motor cortex [34]. This greater stimulus frequency in turn seems to be necessary to maximize benefits to working memory but does not seem necessary to improve long-term memory recall, attention, or set-switching ability. As can be seen in Table 1, heart rate s, and therefore cardiovascular workloads, were similar between ACT and VC, the only plausible explanation that remains for these group differences is the voluntary movement output during VC. The voluntary activation of certain areas of the motor cortex may thus be unique to voluntary exercise or greater in magnitude than the afferent stimulation resulting from ACT and thus benefit the frontal cortex in specific ways.

Differences in performance among executive function tasks, as observed in this study, have been documented [48]. Our results also suggest that different executive functions (e.g., working memory, attention, inhibition, and set-switching), though all mediated by the frontal cortex, may differentially benefit from different modes of exercise.

\section{Future research}

Our future research is to investigate whether exercise can prevent or slow the progression of Alzheimer's disease (AD) in persons with DS. AD is a serious dysfunction of global cognitive control, and adults with DS are at three to five times the risk of early onset of AD compared to the general population $[5,49]$. $\mathrm{AD}$ is a neurocognitive degenerative disease that causes a loss of memory, thinking, and functioning abilities. It is known that as the lifespan of adults with DS increases, the prevalence of AD will rise concomitantly. However, there is not much research in this area [50]. Research in this field can be aimed toward the development of an intervention, as well as prevention of AD in patients with DS. In order to reach that status, there must be more involvement of the DS population into clinical trials so that sufficient data can be collected and analyzed. 
In addition, brain imaging is an important tool and is used in both clinical diagnosis and in the research of $\mathrm{AD}$ in persons with DS. In this research, many different types of imaging techniques are used to determine participant eligibility as well as to test if the imaging can accurately predict the participant's risk of developing AD. These imaging techniques include, but are not limited to, amyloid PET scans, functional MRIs, structural MRIs, and CTs [51]. Each type of imaging will provide a view into the different mechanisms leading to the development of AD. Amyloid PET scans are a type of positron emission tomography in which amyloid plaque is targeted with radioactive tracers to be seen on the image. It is thought that an abnormal amount of these plaques predisposes the participant to developing AD. While this type of imaging is futile in the clinical setting, researchers can choose to look for participants who have this abnormal amount of amyloid plaque but show no symptoms, so that an intervention can be created for those individuals. MRIs and CTs have more impact in the clinical setting because they focus on the structure of the brain. Research has shown that abnormalities in the hippocampus as well as general atrophy of the brain can lead to dementia. Physicians are able to use these imaging tools in order to help determine the etiology of dementia. These types of imaging techniques can also be used in patients with DS in a similar manner. They can help to predict which patients are at a higher risk than others so that an intervention can be put in place earlier.

\section{Practical applications}

Physical activity of any kind will most likely improve cognitive functioning in persons with DS. Often physical activity that the person enjoys (e.g., dancing, gardening, and walking dog) are the types of programs that are sustained. Only $10-12 \%$ of people with DS learn to ride a bicycle and very few learn before 10 years old [52]. However, cycling does allow for fast rates of lower limb movement which is crucial because the increased movement rate may trigger the endogenous release of neurotrophic factors that facilitate the motoric and neural changes that underlie improved motor and cognitive function. To date, our stationary research bicycle is not for sale. One clinical research trial with Parkinson's patients used a tandem bicycle in which the front rider is typical and the back rider had Parkinson's disease. Because the pedals were yoked, the rear rider was pedaling at the same rate as the front rider. However, this may take a lot of practice to perform safely. An alternative recommendation is to be conscious of when you are physically active to try to increase the rate of movement whenever possible. For practical purposes, measure the rate of movement by counting the number of revolutions during a set time period (e.g., 10 s.) and extrapolating to one minute. Heart rate could be palpated or measured by a simple Polar HR monitor. In addition, Rate of Perceived Exertion should be monitored every five minutes especially in special populations such as DS where heart rate responses are different from those of the general population due to chronotropic incompetence [53]. The main goal is to have fun and be safe, and remember if you move faster, you may think faster! 


\section{Author details}

Shannon D. R. Ringenbach*, Simon Holzapfel, Genna M. Mulvey and Sachin Pandya

*Address all correspondence to: Shannon.ringenbach@asu.edu

Arizona State University, Phoenix, AZ, USA

\section{References}

[1] National Down Syndrome Society. (2012). Down syndrome facts. Retrieved from http:// http://www.ndss.org/Down-Syndrome/Down-Syndrome-Facts/

[2] Lanfranchi, S., Jerman, O., Dal Pont, E., Alberti, A., \& Vianello, R. (2010). Executive function in adolescents with Down syndrome. Journal of Intellectual Disability Research, 54(4), 308-319.

[3] Lezak, M. D. (1995). Neuropsychological Assessment. $3^{\text {rd }}$ ed. Oxford University Press: New York.

[4] Das, J. P., Divis, B., Alexander J., Parrila R. K., \& Naglieri, J. A. (1995). Cognitive decline due to aging among persons with Down syndrome. Research in Developmental Disabilities, 16, 461-478.

[5] Rowe, J., Lavender, A., \& Turk, V. (2006). Cognitive executive function in Down's syndrome. British Journal of Clinical Psychology, 45(Pt 1), 5-17.

[6] Baddeley A. (2003). Working memory: Looking back and looking forward. National Review of Neuroscience, 4, 829-839.

[7] Maxwell, J. P., Masters, R. S. W., \& Eves, F. F. (2003). The role of working memory in motor learning and performance. Consciousness and Cognition, 12, 376-402.

[8] Carney, D. P. J., Brown, J. H., \& Henry, L. A. (2013). Executive function in Williams and Down syndrome. Research in Developmental Disabilities, 34(1), 46-55. doi: 10.1016/ j.ridd.2012.07.013

[9] Lanfranchi, W., Cornoldi, C., \& Vianello, R. (2004). Verbal and visuospatial working memory deficits in children with Down syndrome. American Journal on Mental Retardation, 109, 456-466.

[10] Frye, D., Zelazo, P. D., \& Palfai, T. (1995). Theory of mind and rule-based reasoning. Cognitive Development, 10, 483-527.

[11] Davidson, M. C., Amso, D., Anderson, L. C., \& Diamond, A. (2006). Development of cognitive control and executive functions from 4 to 13 years: Evidence from manipu- 
lations of memory, inhibition, and task switching. Neuropsycholotgia, 44(11), 2037-2078.

[12] Periáñez, J.A., Maestú, F., Barceló F., Fernández, A., Amo, C., \& Ortiz-Alonso, T. (2004). Spatiotemporal brain dynamics during preparatory set shifting. Neuroimage, 21, 687-695.

[13] Chapman, R. S. (2003). Language and communication in individuals with Down syndrome. In Abbeduto, L. (Ed). International Review of Research in Mental Retardation: Language and Communication, vol. 27. San Diego, CA, USA, Academic Press, pp. 1-34.

[14] Dykens, E. M., Hodapp, R. M., \& Finucane, B. M. (2000). Genetics and Mental Retardation Syndromes. Paul H. Brookes: New York.

[15] Silverman, W. (2007). Down syndrome: cognitive phenotype. Mental Retardation and Developmental Disabilities Research Reviews, 13, 228-236.

[16] Baldo, J. V., Schwartz, S., Wilkins, D., \& Dronkers, N. F. (2006). Role of frontal versus temporal cortex in verbal fluency as revealed by voxel-based lesion symptom mapping. Journal oft he International Neuropsychological Society, 12, 896-900. doi:10.1017/ S1355617706061078.

[17] Nash, H. M. \& Snowling, M. J. (2008). Semantic and phonological fluency in children with Down syndrome: Atypical organization of language or less efficient retrieval strategies? Cognitive Neuropsychology, 25, 690-703.

[18] Hillman, C. H., Erickson, K. I., \& Kramer, A. F. (2008). Be smart, exercise your heart: Exercise effects on brain and cognition. Nature Review Neuroscience, 9(1), 58-65.

[19] Hillman, C. H., Snook, E. M., \& Jerome, G.J. (2003). Acute cardiovascular exercise and executive control function. International Journal of Psychophysiology, 48(3), 307314.

[20] Buck, S. M., Hillman, C. H., \& Castelli, D. M. (2008). The relation of aerobic fitness to stroop task performance in preadolescent children. Medicine in Science Sports and Exercise, 40(1), 166-172.

[21] Hillman, C. H., Pontifex, M. B., Raine, L. B., Castelli, D. M., Hall, E. E., \& Kramer, A. F. (2009). The effect of acute treadmill walking on cognitive control and academic achievement in preadolescent children. Neuroscience, 159(3), 1044-1054.

[22] Colcombe, S., \& Kramer, A. F. (2003). Fitness effects on the cognitive function of older adults: A meta-analytic study. Psychological Sciences, 14(2), 125-130.

[23] van Uffelen, J. G., Chin, A. P. M. J., Hopman-Rock, M., \& van Mechelen, W. (2008). The effects of exercise on cognition in older adults with and without cognitive decline: A systematic review. Clinical Journal of Sport Medicine, 18, 486-500.

[24] Llorens-Martín, M. V., Rueda, N., Tejeda, G. S., Flórez, J., Trejo, J. L., \& MartínezCué, C. (2010). Effects of voluntary physical exercise on adult hippocampal neuro- 
genesis and behavior of Ts65Dn mice, a model of Down syndrome. Neuroscience, 171(4): 1228-1240.

[25] Andriolo, R. B., El Dib, R. P., Ramos, L., Atallah, A. N., \& da Silva, E.M. (2010). Aerobic exercise training programmes for improving physical and psychosocial health in adults with Down syndrome. Cochrane Database Systems Review, 12(5), CD005176.

[26] Latash, M. L. (1992). Motor control in Down syndrome: The role of adaptation and practice. Journal of Developmental and Physical Disability, 4, 227-261.

[27] Inui, N., Yamanishi, M., \& Tada, S. (1985). Simple reaction times and timing of serial reactions of adolescents with mental retardation, autism, and Down syndrome. Perceptual and Motor Skills, 81(3 Pt 1), 739-745.

[28] Cioni, M., Cocilovo, A., Di Pasquale, F., Araujo, M. B., Siqueira, C. R., \& Bianco, M. (1994). Strength deficit of knee extensor muscles of individuals with Down syndrome from childhood to adolescence. American Journal of Mental Retardation, 99(2), 166-174.

[29] Fernhall, B., Pitetti, K. H., Rimmer, J. H., \& McCubbin, J. A. (1996). Cardiorespiratory capacity of individuals with mental retardation including Down syndrome. Medicine and Science Sports and Exercise, 28(3), 366-371.

[30] Barr, M., \& Shields, N. (2011). Identifying the barriers and facilitators to participation in physical activity for children with Down syndrome. Journal of Intellectual Disability Research, 55(11), 1020-1033.

[31] Petzinger, G. M., Walsh, J. P., Akopian, G., Hogg, E., Abernathy, A., Arevalo, P., Turnquist, P., Vuckovic, M., Fisher, B. E., Togasaki, D. M., \& Jakowec, M, W. (2007). Effects of treadmill exercise on dopaminergic transmission in the 1-methyl-4-phenyl-1, 2, 3, 6-tetrahydropyridine-lesioned mouse model of basal ganglia injury. Journal of Neuroscience, 27, 5291-5300.

[32] Ringenbach, S. D. R., Chen, C. C., Albert, A. R., Lichtsinn, K., \& Alberts, J. L. (2013). Assisted cycle therapy (ACT): Implications for improvements in motor control. In Leitner, M. \& Fuchs, M. (Eds.). Motor Behavior and Control: New Research, Hauppauge, NY: Nova Science Publishers Inc., pp. 103-114.

[33] Fisher, B. E., Petzinger, G. M., Nixon, K., Hogg, E., Bremmer, S., Meshul, C. K., Jakowec, M. W. (2004). Exercise-induced behavioral recovery and neuroplasticity in the 1methyl-4-phenyl-1, 2, 3, 6-tetrahydropyridine- lesioned mouse basal ganglia. Journal of Neurology Research, 77, 378-390.

[34] Alberts, J. L., Linder, S. M., Penko, A. L., Lowe, M. J., \& Phillips, M. (2011). It is not about the bike, it is about the pedaling: Forced exercise and Parkinson disease. Exercise and Sport Science Review, 39(4), 177-186.

[35] Ridgel, A. L., Vitek, J. L., \& Alberts, J. L. (2009). Forced, not voluntary, exercise improves motor function in Parkinson's disease patients. Neurorehabiltation and Neural Repair, 23, 600-608. 
[36] Fernhall, B. O., McCubbin, J. A., Pitetti, K. H., Rintala, P., Rimmer, J. H., Millar, A. L., \& De Silva, A. (2001). Prediction of maximal heart rate in individuals with mental retardation. Medicine and Science in Sports and Exercise, 33(10), 1655-1660.

[37] American College of Sports Medicine. (2013). ACSM's Guidelines for Exercise Testing and Prescription. Baltimore, MD: Lippincott Williams \& Wilkins.

[38] Dunn, D. M., \& Dunn, L. M. (2007). Peabody Picture Vocabulary Test-Fourth Edition. San Antonio, TX: Pearson.

[39] Gabrieli, J. D., Poldrack, R. A., \& Desmond, J. E. (1998). The role of left prefrontal cortex in language and memory. Proceedings of the National Academy of Sciences, 95(3), 906-913.

[40] Weiss, E. M., Siedentopf, C., Hofer, A., Deisenhammer, E. A., Hoptman, M. J., Kremser, C., Golaszewski, S., Felber, S., Fleischhacker, W. W., \& Delazer, M. (2003). Brain activation pattern during a verbal fluency test in healthy male and female volunteers: A functional magnetic resonance imaging study. Neuroscience Letters, 352(3), 191-194.

[41] Fowler, A., Gelman, R., \& Gleitman, L. R. (1994). The course of language learning in children with Down syndrome. Constraints on language acquisition: Studies of atypical children, 91-140.

[42] Hulme, C., \& Mackenzie, S. (1992). Working Memory and Severe Learning Difficulties. Mahwah, NJ: Lawrence Erlbaum Associates, Inc.

[43] Pennington, B. F., Moon, J., Edgin, J., Stedron, J., \& Nadel, L. (2003). The neuropsychology of Down syndrome: Evidence for hippocampal dysfunction. Child development, 74(1), 75-93.

[44] Iverson, G. L., \& Franzen, M. D. (1994). The Recognition Memory Test, digit span, and Knox Cube Test as markers of malingered memory impairment. Assessment, 1(4), 323-334.

[45] Berman, K. F., Ostrem, J. L., Randolph, C., Gold, J., Goldberg, T. E., Coppola, R., Carson, R. E., Herscovitch, P., \& Weinberger, D. R. (1995). Physiological activation of a cortical network during performance of the Wisconsin Card Sorting Test: A positron emission tomography study. Neuropsychologia, 33(8), 1027-1046.

[46] Nelson, H. E. (1976). A modified card sorting test sensitive to frontal lobe defects. Cortex, 12(4), 313-324.

[47] Cohen, J. 1988. Statistical power analysis for the behavioral sciences. Hillsdale, New Jersey: Lawrence Erlbaum Associates

[48] Miyake, A., Friedman, N. P., Emerson, M. J., Witzki, A. H., Howerter, A., \& Wager, T. D. (2000). The unity and diversity of executive functions and their contributions to complex "frontal lobe" tasks: A latent variable analysis. Cognitive Psychology, 41(1), 49-100. 
[49] Wisniewski, K. E., Wisniewski, H. M., \& Wen, G. Y. (1985). Occurrence of neuropathological changes and dementia of Alzheimer's disease in Down's syndrome. Annals of Neurology, 17, 278-282.

[50] Hartley, D., Blumenthal, T., Carrillo, M., DiPaolo G., Esralew, L., Gardiner, K., Granholm, A. C., Igbal, K., Krams, M., Lemere, C., Lott, I., Mobley, W., Ness, S., Nixon, R., Potter, H., Reeves, R., Sabbagh, M., Silverman, W., Silverman, W., Tycko, B., Whitten, M., Wisniewski, T. (2014). Down syndrome and Alzheimer's disease: Common pathways, common goals. Alzheimer's and Dementia, 1-10. doi: 10.1016/j.jalz.2014.10.007. [Epub ahead of print]

[51] Zakzanis, K., Graham, S. J., \& Campbell, Z. (2003). A meta-analysis of structural and functional brain imaging in dementia of the Alzheimer's type: A neuroimaging profile. Neuropsychology Review, 13, 1-18.(1).

[52] Ulrich, D. A., Burghardt, A. R., Lloyd, M., Tiernan, C., \& Hornyak, J. E. (2011). Physical activity benefits of learning to ride a two-wheel bicycle for children with Down syndrome: A randomized trial. Physical Therapy, 91(10), 1463-1477.

[53] Baynard, T., Pitetti, K. H., Guerra, M., \& Fernhall, B. (2004). Heart rate variability at rest and during exercise in persons with Down syndrome. Archives of Physical Medicine and Rehabilitation, 85(8), 1285-1290. 